\title{
Анализ влияния спейсерных слоев на нелинейные искажения вольт-амперных характеристик рНЕМТ на основе соединения GaAIAs/InGaAs
}

\author{
(ㄷ Е.А. Тарасова ${ }^{1}$, С.В. Хазанова ${ }^{1}$, О.Л. Голиков ${ }^{1}$, А.С. Пузанов ${ }^{1}$, С.В. Оболенский ${ }^{1}$, В.Е. Земляков ${ }^{2}$ \\ ${ }^{1}$ Нижегородский государственный университет им. Н.И. Лобачевского, \\ 603600 Нижний Новгород, Россия \\ ${ }^{2}$ Национальный исследовательский университет „Московский институт электронной техники“ (МИЭТ), \\ 124498 Москва, Зеленоград, Росси \\ E-mail: tarasova@rf.unn.ru
}

Поступила в Редакцию 12 апреля 2021 г.

В окончательной редакции 19 апреля 2021 г.

Принята к публикации 19 апреля 2021 г.

Работа посвящена результатам моделирования электрофизических параметров рНЕМТ структур на основе соединения $\mathrm{AlGaAs} / \mathrm{InGaAs} / \mathrm{GaAs}$ с помощью самосогласованного решения уравнения Шредингера и Пуассона. На основании численных расчетов предложен метод анализа нелинейных искажений передаточных вольт-амперных характеристик исследуемых транзисторов. Проведена оценка влияния спейсерных слоев и степени легирования $\delta$-слоя на нелинейность вольт-амперных характеристик.

Ключевые слова: AlGaAs/InGaAs/GaAs pHEMT, нелинейные искажения, спейсерные слои.

DOI: $10.21883 /$ FTP.2021.10.51436.35

\section{1. Введение}

Известно, что применение гетероструктуры $\mathrm{AlGaAs} / \mathrm{InGaAs} / \mathrm{GaAs}$ позволяет создать двумерный электронный газ в канале полевого транзистора с высокой подвижностью электронов (HEMT - High Electron Mobility Transistor) [1]. Так как параметры кристаллической решетки слоев не совпадают, такой транзистор называется псевдоморфным (pHЕМТ). Для увеличения концентрации электронов в канале используется $\delta$-легирование прилегающих к каналу областей широкозонного полупроводника. Для надежного пространственного разделения $\delta$-слоя и квантовой ямы, образующей канал полевого транзистора, а также в других технологических целях (например, снижения микрошероховатости гетерограницы), используют дополнительные тонкие спейсерные слои из нелегированного AlGaAs.

Концентрация электронов и их подвижность в канале полевого транзистора в конечном счете задают его передаточную вольт-амперную характеристику, крутизну передаточной характеристики и старшие производные, определяющие нелинейные свойства полупроводникового прибора. Известно [2], что усилители мощности на основе полевых транзисторов обладают линейностью только при небольших уровнях входного сигнала, при возрастании его мощности появляются нелинейные интермодуляционные искажения, приводящие к искажению выходного сигнала. Таким образом, корректный расчет исходных зависимостей концентрации электронов в канале полевого транзистора с двумерным электронным газом с учетом спейсерных слоев, изменяющих расстояние от поверхности затвора и $\delta$-слоя до квантовой ямы, является важным для оптимизации структуры по крите- рию максимизации коэффициента усиления в линейном режиме.

Величина подвижности электронов рассчитывалась исходя из рассеяния на оптических фононах в 2D-газе [3]. Предполагалось, что наличие спейсерных слоев исключает влияние рассеяния электронов на заряженных примесях. Также учитывался эффект всплеска скорости электронов в коротких структурах $[4,5]$.

\section{2. Объект исследования}

В работе проводится расчет электрофизических параметров рНЕМТ структур на основе соединения $\mathrm{Al}_{0.23} \mathrm{Ga}_{0.77} \mathrm{As} / \mathrm{GaAs} / \mathrm{In}_{0.17} \mathrm{Ga}_{0.83} \mathrm{As}$ со слоевой концентрацией электронов в канале $1.5 \cdot 10^{12}-2.4 \cdot 10^{12} \mathrm{~cm}^{-2}$. Анализировалось влияние как тонких $\mathrm{GaAs}$ спейсерных слоев вокруг слоя с $\delta$-легированием, так и спейсерных слоев вблизи канального слоя. Рассматривалось три варианта структуры: в первом случае не учитывались тонкие спейсерные слои из GaAs около $\delta$-слоя (табл. 1), во втором случае учитывались все спейсерные слои (табл. 2), в третьем варианте проводился учет спейсерных слоев, прилегающих к $\delta$-слою, но не учитывался спейсерный слой из GaAs вблизи канального слоя (табл. 3).

В данной работе для каждого из трех вариантов структур последовательно рассчитывались: зонные диаграммы и концентрация носителей заряда в канале исследуемых структур в зависимости от приложенного к затвору напряжения; ток, протекающий через транзистор в режиме насыщения и интермодуляционные искажения в модельном усилителе для двух вариантов уровня легирования $\delta$-слоя: $5 \cdot 10^{24}$ или $8 \cdot 10^{24} \mathrm{M}^{-3}$. 
Таблица 1. Параметры исследуемой полупроводниковой структуры. Вариант 1. Расстояние от затвора до верхней границы квантовой ямы -24.7 нм

\begin{tabular}{l|c|c}
\hline & Толщина, нм & $\begin{array}{c}\text { Концентрация легирующей } \\
\text { примеси, м }\end{array}$ \\
\hline Барьерный слой $\mathrm{Al}_{0.23} \mathrm{Ga}_{0.77} \mathrm{As}$ & 13 & - \\
$\delta$-слой $\mathrm{Al}_{0.23} \mathrm{Ga}_{0.77} \mathrm{As}$ & 3 & $5 \cdot 10^{24}\left(8 \cdot 10^{24}\right)$ \\
Спейсерный слой $\mathrm{Al}_{0.23} \mathrm{Ga}_{0.77} \mathrm{As}$ & 7 & - \\
Спейсерный слой $\mathrm{GaAs}^{-3}$ & 1.5 & - \\
Канальный слой $\mathrm{In}_{0.23} \mathrm{Ga}_{0.77} \mathrm{As}$ & 10 & - \\
(квантовая яма) & 44 & -
\end{tabular}

Таблица 2. Параметры исследуемой полупроводниковой структуры. Вариант 2. Расстояние от затвора до верхней границы квантовой ямы -25.3 нм

\begin{tabular}{l|c|c}
\hline & Толщина, нм & $\begin{array}{c}\text { Концентрация легирующей } \\
\text { примеси, м }\end{array}$ \\
\hline Барьерный слой $\mathrm{Al}_{0.23} \mathrm{Ga}_{0.77} \mathrm{As}$ & 13 & - \\
Спейсерный слой $\mathrm{GaAs}$ & 0.4 & $5 \cdot 10^{22}$ \\
$\delta$-слой $\mathrm{Al}_{0.23} \mathrm{Ga}_{0.77} \mathrm{As}$ & 3 & $5 \cdot 10^{24}\left(8 \cdot 10^{24}\right)$ \\
Спейсерный слой $\mathrm{GaAs}$ & 0.4 & $5 \cdot 10^{22}$ \\
Спейсерный слой $\mathrm{Al}_{0.23} \mathrm{Ga}_{0.77} \mathrm{As}$ & 7 & - \\
Спейсерный слой $\mathrm{GaAs}$ & 1.5 & - \\
Канальный слой $\mathrm{In} 0.23 \mathrm{Ga} 0.77 \mathrm{As}$ & 10 & - \\
(квантовая яма) & 44 & -
\end{tabular}

Таблица 3. Параметры исследуемой полупроводниковой структуры. Вариант 3. Расстояние от затвора до верхней границы квантовой ямы -23.8 нм

\begin{tabular}{l|c|c}
\hline & Толщина, нм & $\begin{array}{c}\text { Концентрация легирующей } \\
\text { примеси, м }\end{array}$ \\
\hline Барьерный слой $\mathrm{Al}_{0.23} \mathrm{Ga}_{0.77} \mathrm{As}$ & 13 & - \\
Спейсерный слой $\mathrm{GaAs}$ & 0.4 & $5 \cdot 10^{22}$ \\
$\delta$-слой $\mathrm{Al}_{0.23} \mathrm{Ga}_{0.77} \mathrm{As}$ & 3 & $5 \cdot 10^{24}\left(8 \cdot 10^{24}\right)$ \\
Спейсерный слой $\mathrm{GaAs}$ & 0.4 & $5 \cdot 10^{22}$ \\
Спейсерный слой $\mathrm{Al}_{0.23} \mathrm{Ga}_{0.77} \mathrm{As}$ & 7 & - \\
Канальный слой $\mathrm{In}_{0.23} \mathrm{Ga}_{0.77} \mathrm{As}$ & 10 & - \\
(квантовая яма) & 44 & -
\end{tabular}

\section{3. Методика расчетов параметров исследуемой структуры}

С помощью процедуры самосогласованного решения уравнений Шредингера и Пуассона в работе были проведены расчеты энергетического спектра, огибающие волновых функций и профиль распределения концентрации носителей заряда. Используемая численная схема решения основана на дискретизации в координатном пространстве с использованием как однородной, так и неоднородной сетки. Итерационная процедура самосогласованного решения данных уравнений позволяет находить профили потенциала и уровни размерного квантования с высокой точностью (до $10^{-5}$ эВ) $[6,7]$ при плавном изменении внешнего напряжения, приложенного в направлении роста структуры. Таким образом, в работе было получено семейство зонных диаграмм структуры и концентраций носителей заряда при различных напряжениях на затворе транзистора в диапазоне от 0 до $1 \mathrm{~B}$ с шагом $0.05 \mathrm{~B}$. 


\section{4. Результаты расчетов}

Результаты расчетов зонной диаграммы и профиля распределения электронов для исследуемых структур для всех трех вариантов (указанных в табл. 1-3) для концентрации $5 \cdot 10^{24} \mathrm{M}^{-3}$ приведены на рис. 1, для концентрации $8 \cdot 10^{24} \mathrm{M}^{-3}-$ на рис. 2 .

Полученные результаты использовались для аналитического расчета статических передаточных вольт-амперных характеристик (BAX) транзистора на исследуемой гетероструктуре. ВАХ рассчитывались с помощью выражений для расчета скорости носителей заряда в канале и выражения для расчета плотности тока в канале транзистора:

$$
I=W \cdot d g \cdot n \cdot V ; \quad V=\frac{\mu E}{1+\mu E / V_{\mathrm{sat}}},
$$

где $I-$ ток, $W$ - ширина затвора транзистора, $n$ - концентрация носителей заряда, $\mu$ - подвижность носителей заряда, $e-$ заряд электрона, $d g-$ толщина обедненной подзатворной области транзистора,

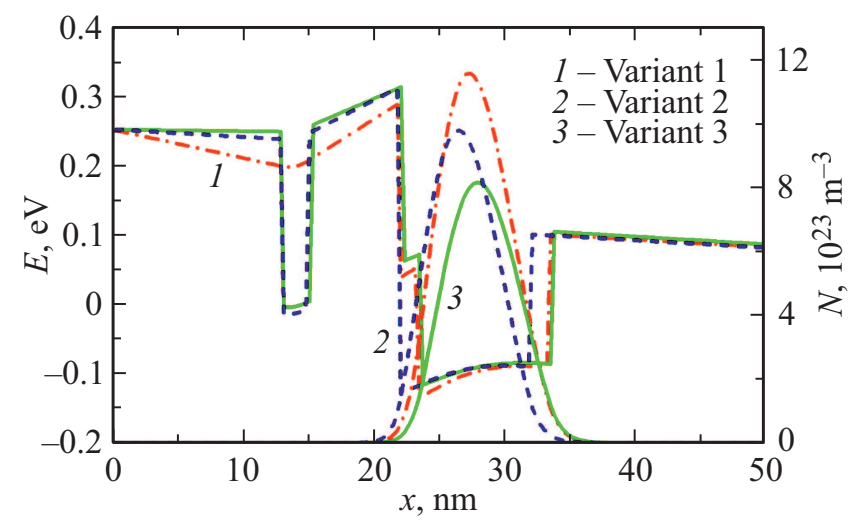

Рис. 1. Результаты расчетов зонной диаграммы и профиля распределения электронов исследуемой структуры для различных комбинаций спейсерных слоев. Степень легирования $\delta$-слоя $5 \cdot 10^{24} \mathrm{M}^{-3}$.

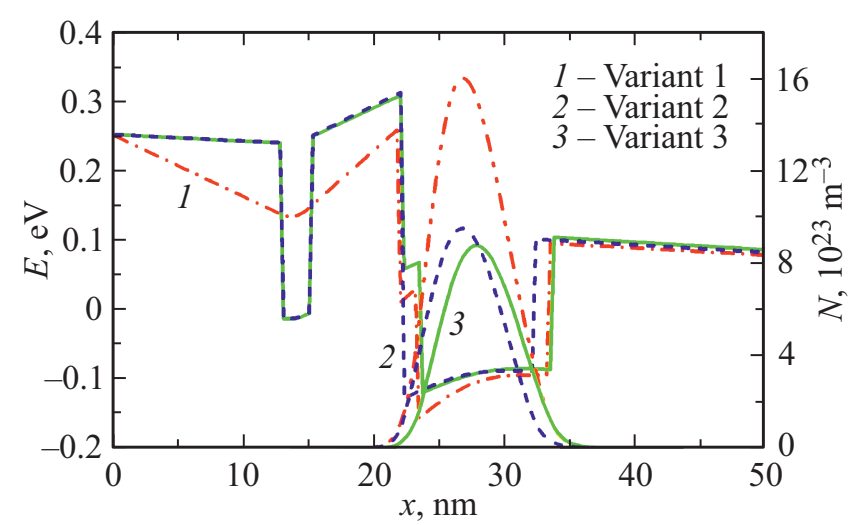

Рис. 2. Результаты расчетов зонной диаграммы и профиля распределения электронов исследуемой структуры для различных комбинаций спейсерных слоев. Степень легирования $\delta$-слоя $8 \cdot 10^{24} \mathrm{M}^{-3}$.

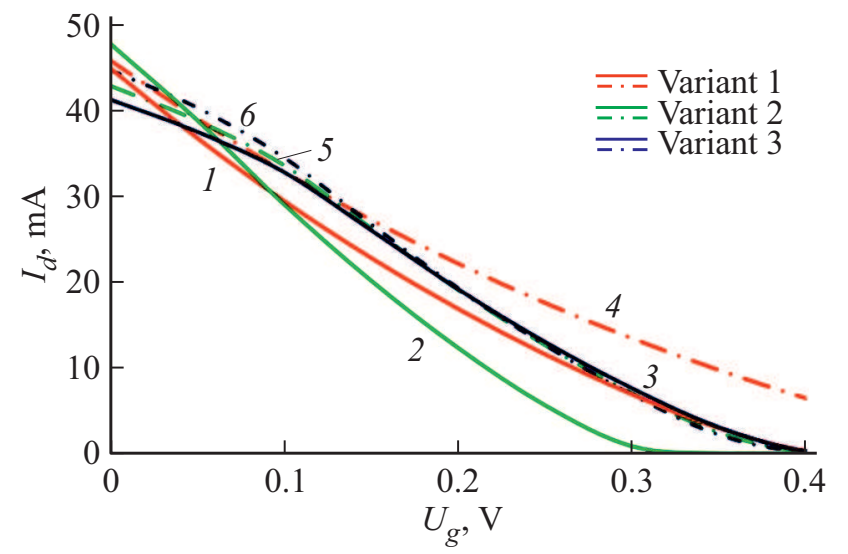

Рис. 3. Результаты аналитического расчета передаточных вольт-амперных характеристик исследуемого рНЕМТ для различных комбинаций спейсерных слоев. Сплошные кривые $(1,2,3)$ соответствуют легированию $\delta$-слоя $5 \cdot 10^{24} \mathrm{M}^{-3}$. Штрихпунктирные кривые $(4,5,6)$ соответствуют легированию $\delta$-слоя $8 \cdot 10^{24} \mathrm{M}^{-3}$. (Цветной вариант рисунка представлен в электронной версии статьи).

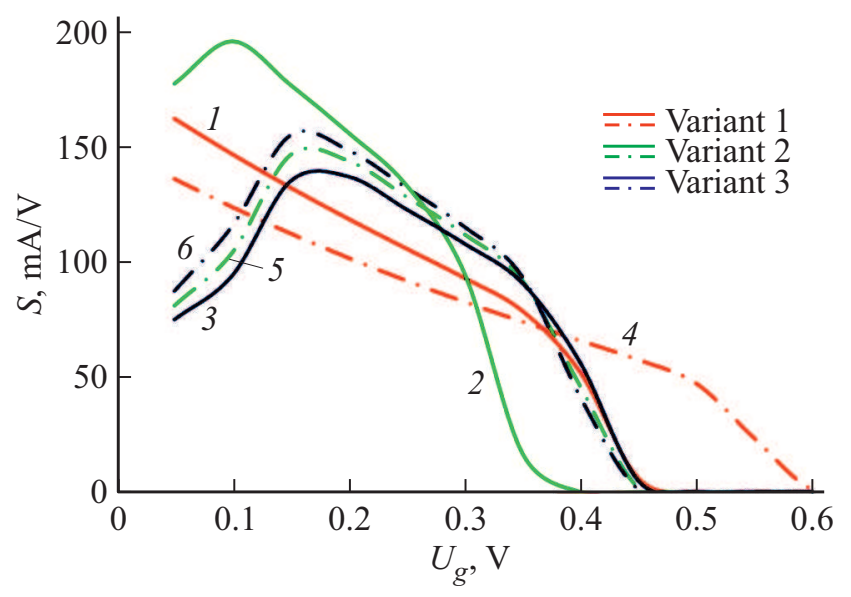

Рис. 4. Результаты аналитического расчета крутизны передаточных вольт-амперных характеристик исследуемого рНЕМТ для степени легирования различных комбинаций спейсерных слоев. Сплошные кривые $(1,2,3)-$ легирование $\delta$-слоя $5 \cdot 10^{24} \mathrm{M}^{-3}$. Штрихпунктирные кривые $(4,5,6)$ - легирование $\delta$-слоя $8 \cdot 10^{24} \mathrm{M}^{-3}$.

$V_{\text {sat }}$ - скорость насыщения. Учитывались полученная из численных расчетов интегральная концентрация носителей заряда в канале исследуемого НЕМТ при различных напряжениях на затворе и эффект всплеска скорости в канале транзистора согласно [4,5]. Зависимость толщины обедненной области в канале транзистора в зависимости от напряжения затвора рассчитывалась согласно [8].

Результаты аналитических расчетов передаточных вольт-амперных характеристик и крутизны BAX для трех вариантов структуры с различной степенью легирования $\delta$-слоя приведены на рис. 3 и 4 соответственно. Различия в полученных результатах объясняются разницей в расстоянии от затвора до верхней границы 
квантовой ямы для каждого из трех рассмотренных вариантов.

\section{5. Методика расчета интермодуляционных искажений}

Известно, что интермодуляционные искажения 3-го порядка существенно ограничивают коэффициент усиления приемников, так как для двух близко расположенных частот $\omega_{1}$ и $\omega_{2}$ только продукты интермодуляции 3-го порядка $2 \omega_{1}-\omega_{2}$ и $2 \omega_{2}-\omega_{1}$ наряду с исходными сигналами проходят выходной фильтр усилителя [2].

Для безынерционного усилителя связь между входом $x(t)$ и выходом $y(t)$ описывается в виде

$$
y(t) \approx \alpha_{1} x(t)+\alpha_{2} x^{2}(t)+\alpha_{3} x^{3}(t)
$$

и представляет собой разложение в ряд Тейлора в окрестности точки смещения [2].

Из приведенного выше выражения видно, что амплитуда выходных продуктов интермодуляции 3-го порядка увеличивается сильнее, чем амплитуда выходного сигнала на основных частотах $\omega_{1}$ и $\omega_{2}$. Таким образом, при повышении амплитуды входного сигнала амплитуда продуктов интермодуляции 3-го порядка в конечном итоге станет равной амплитуде основных тонов на выходе. Уровень, при котором это происходит называется точкой пересечения 3-го порядка (third-order intercept point) по входу (input third-order intercept point - IIP3) и выходу (output third-order intercept point - OIP3) (см. вставку на рис. 5). Нетрудно получить, что

$$
A_{I I P 3}=\sqrt{\frac{3}{4}\left|\frac{\alpha_{1}}{\alpha_{3}}\right|} .
$$

В монографии [2] приведено выражение для точки пересечения 3-го порядка по входу усилителя, построенного по схеме с общим истоком, для квадратичной

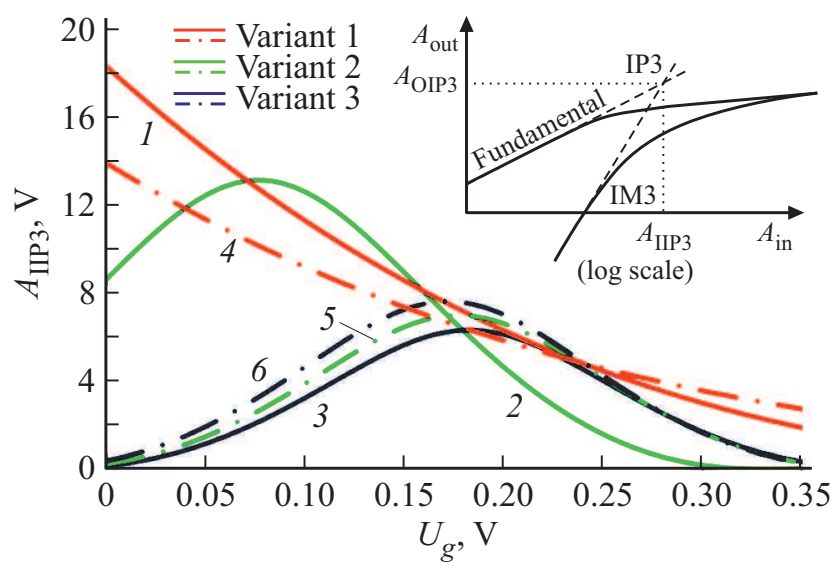

Рис. 5. Результаты расчетов IP3 трех вариантов исследуемой структуры для двух степеней легирования. Сплошные кривые $(1,2,3)$ - легирование $\delta$-слоя $5 \cdot 10^{24} \mathrm{M}^{-3}$. Штрихпунктирные кривые $(4,5,6)$ - легирование $\delta$-слоя $8 \cdot 10^{24} \mathrm{M}^{-3}$. зависимости тока, протекающего через транзистор от напряжения на затворе в виде $I_{D}=K \cdot\left(U_{G}-V_{T H}\right)^{2}$ :

$$
A_{I I P 3}=\frac{(1+R \cdot S)^{2}}{K} \sqrt{\frac{2 \cdot S}{3 \cdot R}}
$$

где $R$ - нагрузочное сопротивление, $S-$ крутизна BAX.

В данной работе указанное выражение расширено на случай, когда зависимость тока, протекающего через транзистор, от напряжения на затворе носит кубический характер

$$
A_{I I P 3}=\sqrt{\left|\frac{8 \cdot S(1+R \cdot S)^{4}}{(1+R \cdot S) \frac{d^{2} S}{d U_{G}^{2}}-3 \cdot R\left(\frac{d S}{d U_{G}}\right)^{2}}\right|} .
$$

Результаты аналитических расчетов интермодуляционных искажений $A_{I I P 3}$ согласно формуле (5) для всех трех вариантов структуры с различной степенью легирования $\delta$-слоя приведены на рис. 5 .

\section{6. Заключение}

Результаты проведенных расчетов подтверждают исходный тезис о значительном влиянии спейсерных слоев на величину нелинейных интермодуляционных искажений в полевых транзисторах с двумерным электронным газом:

1. В случае отсутствия спейсерного слоя GaAs толщиной $0.4 \mathrm{HM} A_{I I P 3}$ монотонно уменьшается с ростом напряжения на затворе транзистора. Увеличение уровня легирования $\delta$-слоя приводит к уменьшению $A_{I I P 3}$ при малых напряжениях на затворе и увеличению $A_{I I P 3}$ при больших напряжениях на затворе.

2. При наличии одного спейсерного слоя GaAs толщиной 0.4 нм $A_{I I P 3}$ имеет максимум в зависимости от напряжения на затворе, при котором влияние нелинейных искажений минимально. Увеличение уровня легирования $\delta$-слоя приводит к уменьшению $A_{I I P 3}$ в точке максимума, но выравниванию относительно динамического диапазона $(\sim 0.2 \mathrm{~B})$.

3. Аналогично при наличии двух спейсерных слоев GaAs толщиной 0.4 нм $A_{I I P 3}$ имеет максимум в зависимости от напряжения на затворе, но при этом увеличение уровня легирования $\delta$-слоя приводит к увеличению $A_{I I P 3}$, т.е. улучшению характеристик транзистора. Также отметим слабую зависимость изменения положения точки максимума от уровня легирования $\delta$-слоя.

Таким образом, по-видимому, оптимальной с точки зрения максимизации коэффициента усиления полевого транзистора в линейном режиме является структура с двумя спейсерными слоями GaAs толщиной 0.4 нм, окружающими $\delta$-слой, так как в этом случае при сравнимой величине $A_{I I P 3}$ с другими вариантами стабильность характеристик значительно выше. 


\title{
Финансирование работы
}

Работа выполнена в рамках базовой части государственного задания, проект 0729-2020-0057.

\section{Конфликт интересов}

Авторы заявляют, что у них нет конфликта интересов.

\section{Список литературы}

[1] T. Mimura. IEEE Trans. Microware Theory and Techniques, 50 (3), 780 (2002).

[2] B. Razavi. RF Microelectronics (Hamilton Printing Company in Castleton, N.Y., 2012).

[3] M. Lundstrom. Fundamentals of carrier transport (Cambridge University Press, 2000).

[4] Ю. Пожела. Физика быстродействующих транзисторов (Вильнюс, Мокслас, 1989).

[5] М. Шур. Современные приборы на основе арсенида галлия (М., Мир, 1991).

[6] С.В. Хазанова, В.Е. Дегтярев, С.В. Тихов, Н.В. Байдусь. ФТП, 49 (1), 53 (2015).

[7] С.В. Хазанова, В.Е. Дегтярев, Н.Н. Григорьева, О.Л. Голиков. Физические и физико-химические основы ионной имплантации (РИУ ННГУ им. Н.И. Лобачевского, 2018).

[8] Е.А. Тарасова, С.В. Оболенский, С.В. Хазанова, Н.Н. Григорьева, О.Л. Голиков, А.Б. Иванов, А.С. Пузанов. ФТП, 54 (9), 968 (2020).

Редактор Г.А. Оганесян

\section{The analysis of the influence of space layers on nonlinear distortions of phemt I-V characteristics based on \\ GaAlAs/InGaAs}

\author{
E.A. Tarasova ${ }^{1}$, S.V. Khazanova ${ }^{1}$, O.L. Golikov' ${ }^{1}$, \\ A.S. Puzanov ${ }^{1}$, S.V. Obolensky ${ }^{1}$, V.E. Zemlyakov ${ }^{2}$ \\ ${ }^{1}$ Lobachevsky State University \\ of Nizhny Novgorod (NNSU), \\ 603950 Nizhny Novgorod, Russia \\ ${ }^{2}$ National Research University \\ of Electronic Technology (MIET), \\ 124498 Moscow, Zelenograd, Russia
}

\begin{abstract}
The work is devoted to the results of modeling of the parameters of pHEMT structures based on the $\mathrm{AlGaAs} / \mathrm{InGaAs} / \mathrm{GaAs}$ compound using a self-consistent solution of the Schrödinger and Poisson equation. Based on numerical calculations, a method for analyzing nonlinear distortions of transfer $\mathrm{I}-\mathrm{V}$ characteristics is proposed. The influence of the spacer layers and the degree of doping of the $\delta$-layer on the nonlinearity of the $\mathrm{I}-\mathrm{V}$ characteristic is estimated.
\end{abstract}

